ENCYCLOPEDDIE Encyclopédie berbère

BERBERE

32 | 2010

32 | Mgild - Mzab

\title{
Microlithes géométriques
}

\section{Roubet}

\section{OpenEdition}

Journals

Édition électronique

URL : https://journals.openedition.org/encyclopedieberbere/596

DOI : 10.4000/encyclopedieberbere.596

ISSN : 2262-7197

\section{Éditeur}

Peeters Publishers

\section{Édition imprimée}

Date de publication : 31 décembre 2010

Pagination : 4997-4999

ISBN : 978-90-429-2369-0

ISSN : 1015-7344

\section{Référence électronique}

C. Roubet, « Microlithes géométriques », Encyclopédie berbère [En ligne], 32 | 2010, document M110, mis en ligne le 06 novembre 2020, consulté le 17 février 2022. URL : http://journals.openedition.org/ encyclopedieberbere/596; DOI : https://doi.org/10.4000/encyclopedieberbere.596

Ce document a été généré automatiquement le 17 février 2022.

(c) Tous droits réservés 


\section{Microlithes géométriques}

\section{Roubet}

1 L'équipement de chasse des derniers chasseurs épipaléolithiques du Maghreb et du Sahara reste assez mal connu. On le suppose constitué de projectiles, représentés par de très petites armatures géométriques. L'usage de l'arc ou d'une hampe n'est chronologiquement attesté que par des représentations rupestres, provenant de régions sahariennes et atlasiques.

2 Microlithe géométrique : ces deux termes, généralement réservés à des armatures pouvant être hypermicrolithiques, désignent des têtes de flèches présentant des extrémités pointues et des bords tranchants ou dentelés; ces armatures de flèches étaient sans doute destinées à " armer » une hampe d'os ou de bois, soit en bout, à la manière d'une fine pointe, soit latéralement, en insérant et fixant (à la glue) et dans une saignée tracée dans la hampe, une ou plusieurs pièces géométriques faisant émerger tranchant et pointes.

3 Ces projectiles se subdivisent en plusieurs types à partir de formes géométriques élémentaires telles que le segment*, le triangle*, le trapèze*, le rhombe*, rarement le rectangle*. Dans la plupart des cas ces types comprennent de nombreux sous-types, définis technologiquement (Tixier 1963, types $\mathrm{n}^{\text {os }} 82$ à 100) (Fig. 1), ils sont caractéristiques de faciès à tendance lamellaire, qui remontent tous au Maghreb à l'Ibéromaurusien. La présence de segments en particulier s'avère presque synchrone de l'apparition de la technique du microburin*. On considère désormais l'apparition du phénomène microlithique ou microlithisation, dans des outillages lithiques lamellaires, comme étant l'une des données fondamentales et constitutives de la rupture technologique existant entre les industries antérieures sur éclats et lames et les nouvelles industries, sur lamelles le plus souvent, qui pénètrent avec l'Homme de Mechta Afalou vers 22000 cal BP., les microlithes géométriques de l'Ibéromauruisien jouent donc un rôle discriminant, accompagnant au Maghreb l'arrivée de l'Epipaléolithique.

4 Le segment présent dés le début de l'Ibéromaurusien, se maintient jusqu'au Néolithique. Ce géométrique n'est donc pas un bon repère. Le triangle, comme $\mathrm{S}$. Hachi 
l'a montré à Afalou Bou Rhummel (2003), s'inscrit dans les contextes ibéromaurusiens postérieurs $18000 \mathrm{cal}$ BP. L'éventail morphologique du triangle est peu ouvert.

Plus tard, avec l'installation de la culture capsienne holocène, triangles et trapèzes prolifèrent et leurs formes s'allongent, évoluent et se multiplient.

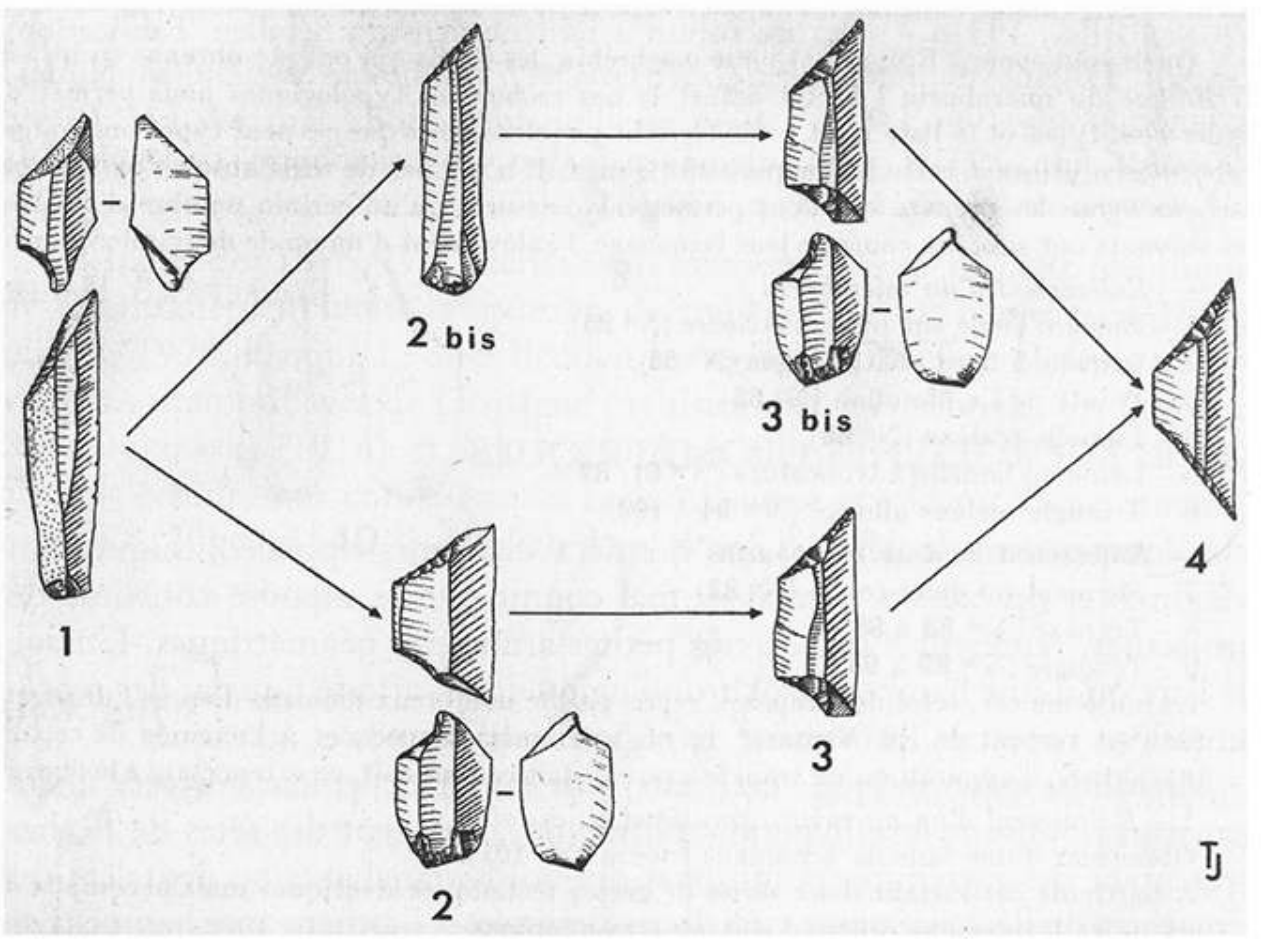

FABRICATION D'UN TRAPÈZE, D'APRÈS J. TIXIER 1963, P. $142: 1$ : LAMELLE À PIQUANT-TRIÈDRE SURMONTÉE DE SON MICROBURIN (VU SUR LES DEUX FACES) ; 2 : MICROBURIN PROXIMAL (VU SUR LES DEUX FACES) SURMONTÉ D'UN TRAPÈZE PORTANT UN BORD OBLIQUE DROIT, À RETOUCHE ABRUPTE

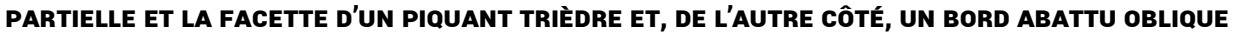
gAUCHE, CONSTITUÉ DES MÊMES CARACTÉRISTIQUES. 2bis : LAMELLE À TRONCATURE DISTALE OBLIQUE. 3 : TRAPÈZE AYANT DEUX TRONCATURES OBLIQUES RETOUCHÉES, OBLITÉRANT TOTALEMENT LES FACETTES DES PIQUANT-TRIÈDRES. 3bis : MICROBURIN PROXIMAL ET TRAPÈZE À TRONCATURES PARTiellement Retouchées. 4 : trapèze ACheVÉ.

Dès le Capsien typique, mais surtout dans le Capsien supérieur, ces formes sont abondantes (Inizan et al. 1995). Le Capsien supérieur représente alors le faciès qui excella dans l'élaboration d'armatures effilées, composites, supposées -pour certaines d'entre elles -avoir servi d'instruments spéciaux en relation avec des pratiques cultuelles telles les scarifications et les tatouages.

7 A l'occasion d'un essai de régionalisation des faciès épipaléolithiques, G. Camps (1974) s'est appuyé sur des données quantitatives et morphologiques du groupe des microlithes géométriques (provenant de travaux fiables et datés) pour distinguer des nuances, des tendances parfois, à l'intérieur du faciès Capsien supérieur, que l'écart des fourchettes chronologiques d'alors ne permettait pas d'établir. Et ce point de vue reste valable. Il existe bien des provinces privilégiant notamment certains microlithes (ex. contextes Tébéssien*, Sétifien* etc.). Le sujet reste à approfondir.

8 Le trait comportemental le plus important que ces armatures géométriques mettent indirectement en évidence concerne les changements économique et technologique survenus dans l'acquisition et la collecte de subsistances animales (terrestres et marines) et végétale. Ces changements fondamentaux caractérisent l'Epipaléolithique. 
On retiendra aussi que lorsque ces géométriques sont absents (cas du Néolithique de Méniet*), nous ne savons pas par quoi ils furent remplacés pour comprendre les changements économiques survenus.

\section{BIBLIOGRAPHIE}

Les ouvrages généraux récents qui mentionnent ce vocabulaire pourraient être utilement consultés.

Dictionnaire de la Préhistoire, sous la direction de A. Leroi-Gourhan. Presses Universitaires de France, 1988.

Dictionnaire de la Préhistoire. Préface de Y. Coppens. Encyclopaedia Universalis. 1999.

La Préhistoire : Histoire et Dictionnaire, sous la direction de D. Vialou. Laffont, Bouquins, 2004. CAMPS G., 1974 - Les civilisations préhistoriques de l'Afrique du Nord et du Sahara. Doin, France. HACHI S., 2003 - Les cultures de l'Homme de Mechta-Afalou. Le gisement d'Afalou Bou Rhummel (massif des Babors, Algérie). Les niveaux supérieurs 13 000-11 000 BP. CNRPAH, Alger.

INIZAN M.-L., REDURON M., ROCHE H., TIXIER J., 1995 - Technologie de la pierre taillée. Préhistoire de la pierre taillée. Tome IV, CNRS, Université Paris-X Nanterre, 199 p.

TIXIER J., 1963 - Typologie de l'Epipaléolithique du Maghreb. Paris, AMG, Mémoire II du CRAPE.

\section{INDEX}

Mots-clés : Chasse, Préhistoire, Technologie 